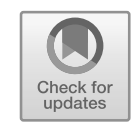

\title{
16 Schlussbemerkung
}

Dieses Buch ist das Ergebnis von mehr als 30 Jahren Forschung mit und über sozialwissenschaftliche Surveys. Diese langjährige Beschäftigung mit Surveys legt einige Schlussfolgerungen nahe:

1. Surveys besitzen großes wissenschaftliches Potential, das sowohl aus methodologischer Unkenntnis als auch aus Arbeitsunwilligkeit oft falsch genutzt wird.

2. Durch die amateurhafte und übermäßige Nutzung von Befragungen - nicht zuletzt durch die universitäre Forschung - werden die sozialen Voraussetzungen der Anwendbarkeit von Surveys der allgemeinen Bevölkerung gefährdet.

3. Surveys besitzen inhärente methodische Probleme, die prinzipiell nicht lösbar sind. Dazu gehören wesentlich die menschlichen kognitiven Grenzen.

4. Will man valide Daten über das Verhalten von Personen, muss man das Verhalten von Personen im Zeitablauf beobachten und nicht durch Befragung erschließen.

Befragungen der allgemeinen Bevölkerung stoßen zunehmend auf Probleme, vor allem, weil der Sinn der Befragung immer schwerer vermittelbar ist. ${ }^{1}$ Prinzipielle Veränderungen der Datenerhebungspraxis erscheinen daher langfristig unvermeidlich.

Surveys werden vor allem dadurch von Bedeutung bleiben, dass Befragungen auf der Ebene von Individuen mit Daten aus anderen Quellen verknüpft werden können. Neben prozessproduzierten Daten wie Mobilitäts-, Transaktions- und Sensordaten sind dies vor allem administrative Daten. $\mathrm{Zu}$ diesen neuen Datenquellen gehören auch Biomarker aller Art. ${ }^{2}$

Surveys werden bei solchen Forschungsstrategien vor allem für die Gewinnung der Mitglieder einer Zufallsstichprobe zur Zusammenarbeit mit dem Untersuchungsteam verwendet: Befragungsdaten sind hierbei nur ein kleiner Teil der Datenerhebung.

1 Zu ähnlichen Schlußfolgerungen siehe Couper (2013), Stern/Bilgen/Dillman (2014), Singer (2016), Miller (2017) sowie Robert Groves in einem Interview (Habermann/Kennedy/Lahir 2017).

2 Einzelheiten zu technischen und juristischen Problemen der Datenverknüpfung finden sich bei Schnell (2018a); Hinweise zur Rolle von Biomarkern und deren Erhebungen in Surveys finden sich bei Schnell (2015b) und Harris/Schorpp (2018). 